\title{
Pareto optimality in multilayer network growth
}

Article

Accepted Version

Santoro, A., Latora, V., Nicosia, G. and Nicosia, V. (2018) Pareto optimality in multilayer network growth. Physical Review Letters, 121 (12). 128302. ISSN 0031-9007 doi: https://doi.org/10.1103/PhysRevLett.121.128302 Available at https://centaur.reading.ac.uk/78736/

It is advisable to refer to the publisher's version if you intend to cite from the work. See Guidance on citing.

To link to this article DOI: http://dx.doi.org/10.1103/PhysRevLett.121.128302

Publisher: American Physical Society

All outputs in CentAUR are protected by Intellectual Property Rights law, including copyright law. Copyright and IPR is retained by the creators or other copyright holders. Terms and conditions for use of this material are defined in the End User Agreement.

\section{www.reading.ac.uk/centaur}

\section{CentAUR}

Central Archive at the University of Reading

Reading's research outputs online 


\title{
Pareto optimality in multilayer network growth
}

\author{
Andrea Santoro, ${ }^{1,2}$ Vito Latora, ${ }^{1,3}$ Giuseppe Nicosia, ${ }^{4,5}$ and Vincenzo Nicosia ${ }^{1}$ \\ ${ }^{1}$ School of Mathematical Sciences, Queen Mary University of London, Mile End Road, E1 4NS, London (UK) \\ ${ }^{2}$ Scuola Superiore di Catania, Università di Catania, Via Valdisavoia 9, 95125, Catania (Italy) \\ ${ }^{3}$ Dipartimento di Fisica ed Astronomia, Università di Catania and INFN, I-95123 Catania, (Italy) \\ ${ }^{4}$ Dipartimento di Matematica ed Informatica, Università di Catania, V.le A. Doria 6, 95125, Catania (Italy) \\ ${ }^{5}$ Department of Computer Science, University of Reading, Whiteknights, RG6 6AF Reading, (UK)
}

(Dated: July 20, 2018)

\begin{abstract}
We model the formation of multi-layer transportation networks as a multi-objective optimization process, where service providers compete for passengers, and the creation of routes is determined by a multi-objective cost function encoding a trade-off between efficiency and competition. The resulting model reproduces well real-world systems as diverse as airplane, train and bus networks, thus suggesting that such systems are indeed compatible with the proposed local optimization mechanisms. In the specific case of airline transportation systems, we show that the networks of routes operated by each company are placed very close to the theoretical Pareto front in the efficiency-competition plane, and that most of the largest carriers of a continent belong to the corresponding Pareto front. Our results shed light on the fundamental role played by multi-objective optimization principles in shaping the structure of large-scale multilayer transportation systems, and provide novel insights to service providers on the strategies for the smart selection of novel routes.
\end{abstract}

PACS numbers:

The interactions among the basic units of many natural and man-made systems, including living organisms, ecosystems, societies, cities, and transportation systems are well described by complex networks [1-5]. Often these systems are subject to different types of concurrent, and sometimes competing, constraints and objectives, such as the availability of energy and resources, or the overall efficiency of the resulting structure. It is therefore reasonable to assume that the systems that we observe today are the result of a delicate balance between contrasting forces, which can be modeled by means of an underlying optimization process under a set of constraints [6-10]. For instance, the emergence of scale-free networks can be explained by simple optimization mechanisms [11-15], while it has been found that many of the properties of biological networks result from the simultaneous optimization of several concurrent cost functions [16-25]. However, multi-objective optimization has not yet been linked to the most recent advances in network science, based on multilayer network representations of real-world systems [26-29]. Recent studies have shown that the presence of many intertwined layers in a network is responsible for the emergence of novel physical phenomena including abrupt cascading failures [3032], superdiffusion [33], explosive synchronisation [34], and the appearance of new dynamical phases in opinion formation [35, 36] and in epidemic processes [37, 38]. Moreover, multiplexity can have an impact on practical problems such as air traffic management [39-41] and epidemic containment $[42,43]$. As a result, understanding how multi-layer networks evolve [44-46] is becoming of central importance in various fields.

In this Letter we propose a model of multilayer network growth in which the formation of links at each layer is the result of a local Multi-Objective Optimization (MOO), i.e., a process where two or more objective (cost) functions, often in conflict to each other, have to be simultaneously minimized or maximized. Within this framework, the concept of Pareto optimality naturally arises. By introducing the dominance strict partial order [3], the solution of a MOO problem consists of a set of non-dominated or Pareto-optimal points in the solution space. Intuitively, these points represent those solutions for which no improvement can be achieved in one objective function without hindering the other objective functions. The collection of non-dominated points constitutes the Pareto surface or Pareto front (PF) [4, 49].
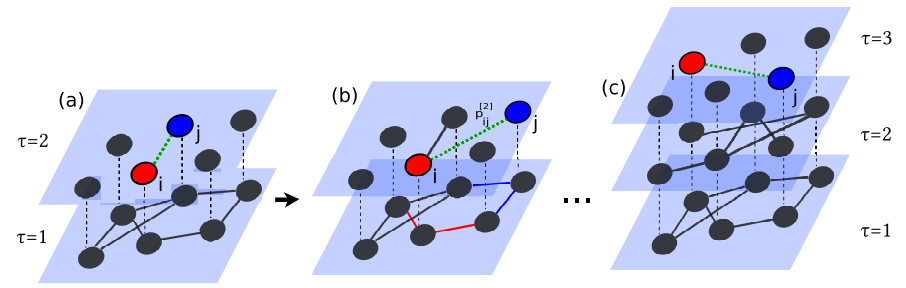

FIG. 1: (Color online) Illustration of the airline growth model. (a) At time $\tau=2$ a new layer arrives with $K^{[2]}$ links to be placed, and the first edge is placed uniformly at random among all possible pairs of nodes. (b) The remaining $K^{[2]}-1$ links are placed according to the probability $p_{i j}^{[2]}$ in Eq. (1). (c) The same procedure is repeated for each layer $\tau$ until a multiplex with $M$ layers is obtained.

The Multiplex Multi-Objective Optimization (MMOO) model we propose is inspired by the observation that the formation of edges in many real-world transportation networks $[50,51]$ is often subject to concurrent spatial and economical constraints [52-54]. 


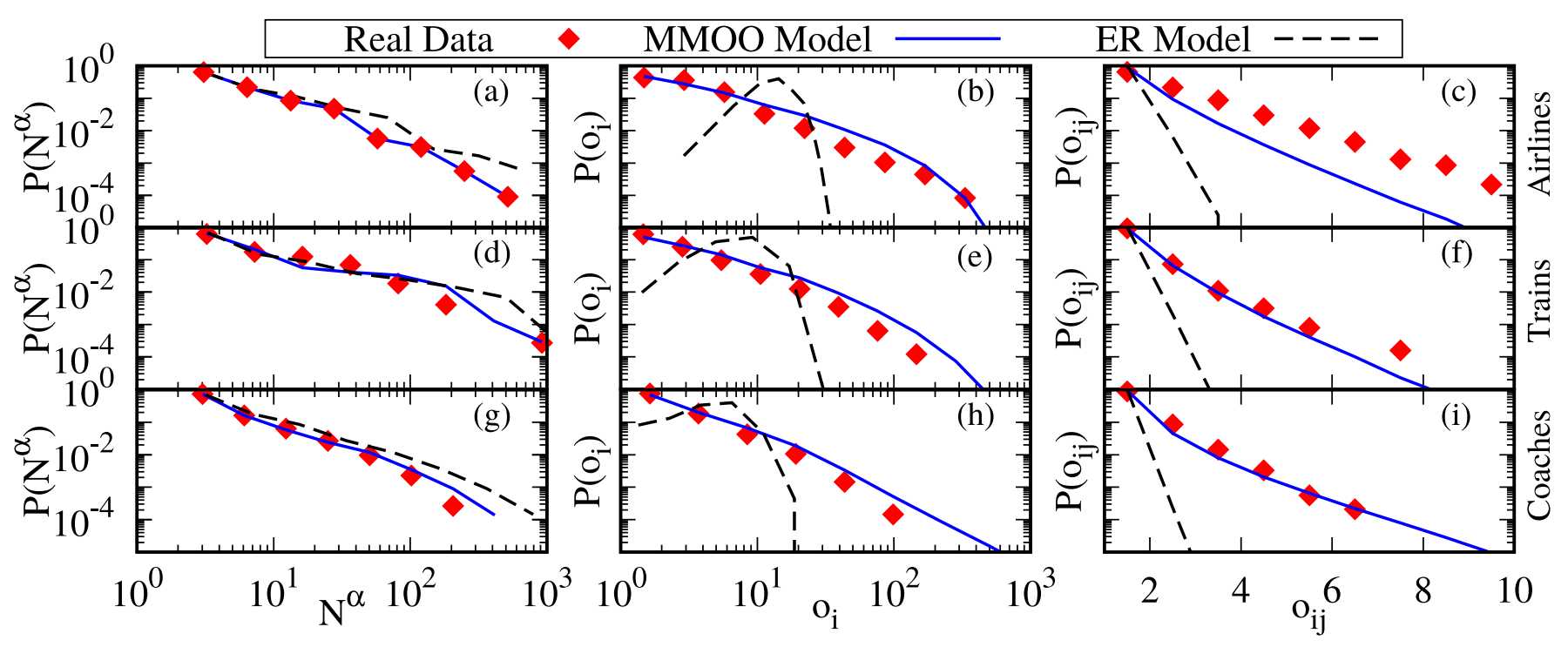

FIG. 2: (Color online) Distributions of layer activity $N^{[\alpha]}$ (left column), node total degree $o_{i}$ (middle column) and edge overlap $o_{i j}$ (right column). The multiplex networks (red diamonds) of North America airlines (top row), UK train services (middle row) and UK coach services are compared to the corresponding multiplex networks generated by the MMOO model (solid blue lines) and to multiplex networks whose layers are Erdös-Rényi graphs [67] (dashed lines). The results shown are averaged over $10^{3}$ realizations (standard deviations are indistinguishable from the symbols).

On the one hand there is the tendency to accumulate edges around nodes that are already well-connected, in order to exploit the economy of scale associated to hubs. On the other hand, each service provider usually tends to minimize the competition with other existing service providers. We show that, by combining these two mechanisms, the MMOO model is able to reproduce quite accurately the structural features of three largescale multiplex transportation systems, namely, the UK railway network, the UK coach network, and the six continental air transportation networks $[7,55,56,58]$. The MMOO model provides a reasonable explanation for the emergence of highly-optimized heterogeneous multiplex networks.

\section{Multiplex Multi-Objective Optimisation Model} (MMOO). - Let us consider a multiplex transportation network with $N$ nodes and $M$ layers, where nodes represent locations and layers represent service providers, e.g. airline, train or bus companies. Each layer is the graph of routes operated by one of the service providers. The network can be described by a set of adjacency matrices $\left\{A^{[1]}, A^{[2]}, \ldots, A^{[M]}\right\} \in \mathbb{R}^{N \times N \times M}$, where the entry $a_{i j}^{[\tau]}$ is equal to 1 if $i$ and $j$ are connected by a link at layer $\tau$ (meaning that provider $\tau$, with $\tau=1,2, \ldots M$ operates a route between location $i$ and $j$ ), while $a_{i j}^{[\tau]}=0$ otherwise. We denote by $k_{i}^{[\tau]}=\sum_{j} a_{i j}^{[\tau]}$ the degree of a node $i$ at the layer $\tau$, and by $K^{[\tau]}=\frac{1}{2} \sum_{i} k_{i}^{[\tau]}$ the total number of links of layer $\tau$. An important multiplex property of a node $i$ is the overlapping (or total) degree $o_{i}=\sum_{\tau} k_{i}^{[\tau]}=\sum_{j} o_{i j}$, namely the total number of edges incident on node $i$ at any of the layers of the multiplex [59], where $o_{i j}=\sum_{\tau} a_{i j}^{[\tau]}$ is the overlap of edge $(i, j)[59,60]$, that is the number of layers at which $i$ and $j$ are connected by an edge.

In the model we assume that service providers join the system one after the other, each one with a predetermined number of routes that they can operate. This means that the multi-layer network acquires a new layer at each (discrete) time step $\tau$. When the layer joins the system, the new provider tries to place its routes in order to maximize its profit. To this end, a provider would prefer to have access to as many potential customers as possible (i.e., to connect locations with large population), whilst minimizing the competition with other providers (i.e., to avoid to operate a route if it is already operated by other providers). In order to mimic these two competing drives, we set the probability to create an edge between node $i$ and node $j$ at the new layer $\tau$ as:

$$
p_{i j}^{[\tau]} \propto \frac{o_{i}^{[\tau-1]} o_{j}^{[\tau-1]}+c_{1}}{o_{i j}^{[\tau-1]}+c_{2}} \quad \tau=2, \ldots, M
$$

where $o_{i}^{[\tau-1]}$ and $o_{i j}^{[\tau-1]}$ are respectively the overlapping degree of node $i$ and the edge overlap of $(i, j)$ at time $\tau-1$. The non-negative constants $c_{1}$ and $c_{2}$ allow a nonzero probability to create a new edge to a node that is isolated at all the existing layers. The rationale behind Eq. (1) is that the overlapping degree $o_{i}$ of node $i$ can be used as a proxy of the population living at that location. Hence, in the same spirit of the "gravity model" [61, 62], creating a link between node $i$ and node $j$ with a prob- 

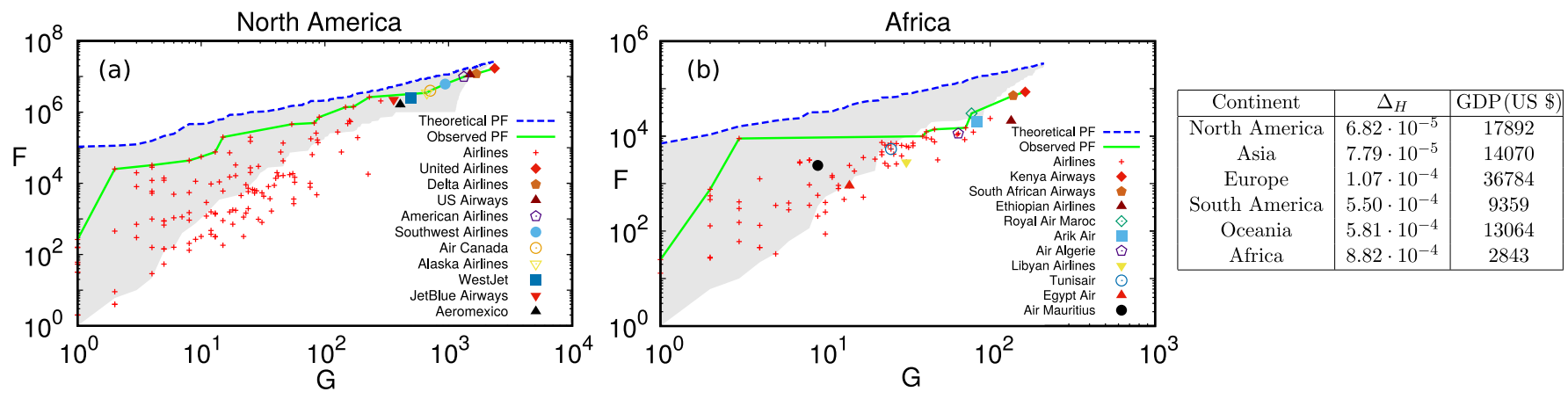

FIG. 3: (Color online) Observed (solid green line) and theoretical (dashed blue line) Pareto fronts for the continental airline networks of (a) North America and (b) Africa. The top ten airlines by number of passengers in 2013 are highlighted. For each system, the theoretical Pareto front was obtained as the non-dominated points of $10^{5}$ realizations of the MMOO model (the range of variability of the simulations is indicated by the shaded gray region). The potential improvement attainable by a system in the $F-G$ plane is measured by the normalized relative hypervolume $\Delta_{H}$, where smaller values of $\Delta_{H}$ correspond to more optimized networks. The ranking of continents by $\Delta_{H}$ is reported in the table together with the economical performance of each continent, as measured by the average GPD per capita.

ability proportional to the product $o_{i} o_{j}$ will increase the chances for a provider to access a large set of customers. Similarly, by requiring that $p_{i j}^{[\tau]}$ is inversely proportional to the edge overlap $o_{i j}^{[\tau-1]}$ we discourage the creation of a new route between two locations if they are already served by a large number of other providers, thus modeling the tendency of providers to avoid competition. The two competing mechanisms we propose can be formalized as a MOO problem:

$$
\left\{\begin{array}{l}
\max \mathbf{F} \\
\min \mathbf{G}
\end{array}=\left\{\begin{array}{l}
F^{[\tau]}=\sum_{i, j: a_{i j}^{[\tau]}=1}\left(o_{i} o_{j}+c_{1}\right) \\
G^{[\tau]}=\sum_{i, j: a_{i j}^{[\tau]}=1}\left(o_{i j}+c_{2}\right)
\end{array}\right.\right.
$$

where the efficiency function $F^{[\tau]}$ accounts for the number of potential customers, while $G^{[\tau]}$ measures the competition due to route overlaps.

The MMOO model is illustrated in Fig. 1. The first layer is a connected random graph with $K^{[1]}$ edges. At each step $\tau$, with $\tau=2, \ldots, M$, a new layer is created. The first of the $K^{[\tau]}$ edges of the new layer is placed uniformly at random among the $\left(\begin{array}{c}N \\ 2\end{array}\right)$ possible edges. In order to obtain a connected network, the remaining $K^{[\tau]}-1$ links are created according to the probability in Eq. (1), yet ensuring that one of the two endpoints of the selected edge belongs to the connected component at that layer. The total number of links at each of the $M$ layers are external parameters of the model. Although considering the routes of each company as fixed over time may look like an unrealistic oversimplification, in all the systems we have considered providers update their network of routes normally at a very slow rate, which justify our assumption to consider the routes on each layer as quasi-static.
In fact, rearranging a set of train services or flights entails substantial logistic and economic investments, since railway licenses and airport-slots are normally allocated over time scales of several years.

Results. - We have used the MMOO model to reproduce the structure of three different multiplex transportation systems. The first data set includes six multiplex air transportation networks, each representing the airline routes operated in a continent. Each network has between 200 and 1000 nodes (airports) and between 35 and 200 layers (carriers) [7]. We have constructed the other two data sets respectively from the UK national railway timetable (41 companies operating over about 1600 stations) and from the UK national coach timetable (1207 companies and over 12000 coach stations). See Ref. [58] for details. For each network, we generated $10^{3}$ independent permutations of the sequence $\left\{K^{[1]}, K^{[2]}, \ldots, K^{[M]}\right\}$ of the total number of links at each layer in the data set. Then, for each permutation, we ran 50 independent realizations of the model. In our simulations we used a Metropolis-Hastings algorithm [63] to sample form the distribution in Eq. (1). In Fig. 2 we report the distributions of layer activity $N^{[\alpha]}$ (number of non-isolated nodes at each layer), total node degree $o_{i}$, and edge overlap $o_{i j}$ of the multiplex networks obtained with the MMOO model, where we set $c_{1}=c_{2}=1$. The two-sample Cramer-von-Mises statistical test [64] provides convincing evidence that the synthetic distributions are compatible with the original ones ( $p$-value $<0.01$, except for panel (c), where $p<0.2$ ). It is worth noticing that the MMOO model naturally reproduces the heterogeneous distribution of node total degree and the decreasing exponential behavior of the edge overlap $o_{i j}$, which respectively mirror the heavy-tailed distribution of city size $[65,66]$ and the tendency of service providers to reduce the competition on single routes [56]. It is also 
possible to fine-tune the values of $c_{1}$ and $c_{2}$ in Eq. (1) in order to accurately reproduce also other structural properties of the three transportation networks, such as the distributions of node activity $B_{i}$ (number of layers at which node $i$ is not isolated) and the pattern of pairwise inter-layer correlations. See Ref. [58] for more details.

Pareto fronts and system efficiency. - By considering the multi-objective optimization framework formally defined in Eq. (2), it is possible to compare providers by looking at their position in the efficiency-competition plane defined by the two functions $F$ and $G$ and shown in Fig. 3. We focused on the air transportation networks and extracted from the empirical data the observed Pareto front of each continental network, i.e., the set of all the non-dominated points in the $F-G$ plane. Surprisingly, we found that most of the Pareto-optimal points correspond to the most important companies in the continent (e.g., flagship, mainline, and large low-cost carriers), and in particular with those carrying the largest number of passengers.

In order to quantify the potential improvement attainable by a system in the $F-G$ plane, we used a multiobjective optimization algorithm [5] to generate $10^{5}$ synthetic multiplex networks for each continent. We then computed the so-called theoretical Pareto front, consisting of the Pareto-optimal points resulting from all the simulations, reported in Fig. 3 as a dashed blue line. The closer the observed PF is to the theoretical PF, the better the system approaches the best possible solution in the $F-G$ plane. Interestingly, the observed $\mathrm{PF}$ of the North American airline network is relatively closer to its theoretical PF, while for the African airlines we observe a larger gap between the two curves. This means that, on average, the African airlines may obtain a greater improvement in the $F-G$ plane than North American companies. A quantitative way to associate a number to a Pareto Front $\mathcal{P}$ is by means of the hypervolume indicator $I_{H}(\mathcal{P})$, that is the Lebesgue measure of the union of the rectangles defined by each point in the front $\mathcal{P}$ and a reference point $[1,2,58]$. The distance between an observed $\mathrm{PF}, \mathcal{P}^{\text {obs }}$, and the corresponding theoretical PF, $\mathcal{P}^{\text {th }}$, can be quantified through the relative normalized hypervolume $\Delta_{H}=\left|I_{H_{\mathcal{P} \text { obs }}}-I_{H_{\mathcal{P} \text { th }}}\right| /\left(I_{H_{\mathcal{P} \text { th }}} K\right)$. By dividing the relative hypervolume difference by the total number of routes $K$, it is possible to compare the level of potential improvement of two multiplex networks with respect to their corresponding theoretical PF. We argue that the value of $\Delta_{H}$ can be used as a proxy of the technological advancement of a transportation system, with smaller values of $\Delta_{H}$ indicating more optimized configurations. The Table in Fig. 3 reports the ranking of the continents induced by $\Delta_{H}$, where North America and Asia lead the pack, while Africa is lagging behind. Interestingly, that ranking is positively correlated with the ranking induced by continental GDP per capita [71] (Kendall's $\tau_{b}=0.6$,

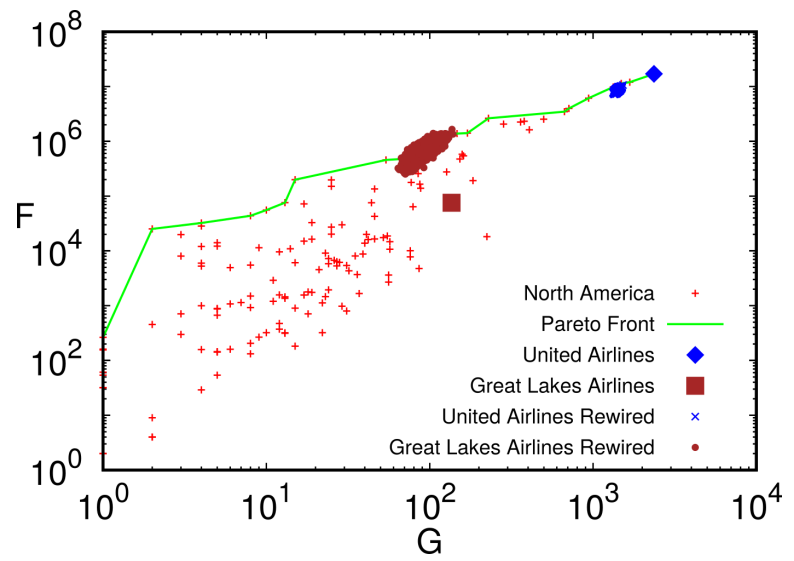

FIG. 4: (Color online) Reshaping the routes of two North American airlines in the $F-G$ plane. United Airlines, a Paretooptimal company (blue diamonds), cannot be improved any further by our model, while Great Lakes Airlines (brown squares) can potentially get much closer to the Pareto front.

$\left.p \approx 9 \cdot 10^{-2}\right)$.

Finally, we show that our model can in principle be used by new companies entering the market, as a guide to place their routes in the most effective way. We run simulations of a slightly different version of the model, where all the layers are fixed and identical to the observed ones except for one of them, which represents a new service provider. The last layer is constructed according to Eq. (1). As shown in Fig. 4, we found that in general the companies lying on the observed Pareto front cannot substantially improve their position in the $F-G$ plane, meaning that their routes have evolved over time according to an effective optimization process. Conversely, our model is able to improve the position in the $F-G$ plane of sub-optimal and non-optimal airlines.

Conclusions. - The introduction of multi-objective optimization principles in the modeling of multi-layer systems allows to obtain simple, effective explanations for the evolution of real-world transportation networks. In particular, the systematic exploration of the possible local improvements in the efficiency-competition plane at the level of single carriers, that we have used here to characterize the technological advancement of a continent and the effectiveness of the network of single carriers, can be employed in practice to inform the placement of new routes, and to compare alternative expansion strategies. The proposed methodology can be readily applied to any system whose multi-layer structure is the result of the interactions between two or more conflicting objective functions, paving the way to a more accurate characterization of many different natural and man-made complex systems.

V. L. acknowledges support from the EPSRC project $\mathrm{EP} / \mathrm{N} 013492 / 1$. 
[1] R. Albert, A. L. Barabási, Rev. Mod. Phys. 74, 47 (2002).

[2] M. E. J. Newman, SIAM Rev. 45, 167 (2003).

[3] S. Boccaletti, V. Latora, Y. Moreno, M. Chavez, D.-U. Hwang, Phys. Rep. 424, 175 (2006).

[4] M. E. J. Newman, Networks: An Introduction (Oxford University Press, New York, 2010).

[5] V. Latora, V. Nicosia, G. Russo, Complex Networks: Principles, Methods and Applications (Cambridge University Press, Cambridge, England, 2017).

[6] R. F. Cancho, R. V. Solé, Lect. Notes Phys. 625, 114-125 (2003).

[7] M. T. Gastner, M. E. J. Newman, The Eur. Phys. J. B 49, 2 (2006).

[8] M. Barthélemy, A. Flammini, J. Stat. Mech. L07002 (2006).

[9] M. Barthélemy, A. Flammini, Phys. Rev. Lett. 100, 138702 (2008).

[10] R. Louf, P. Jensen, M. Barthélemy, Proc. Natl. Acad. Sci. U.S.A. 110, 22 (2013).

[11] A. Fabrikant, E. Koutsoupias, C. Papadimitriou, Lecture Notes in Computer Science 2380, 110-122 (2002).

[12] R. M. D'Souza, C. Borgs, J. T. Chayes, N. Berger, R. D. Kleinberg, Proc. Natl Acad. Sci. USA 104, 6112-6117 (2007).

[13] S. Valverde, R. F. Cancho, R. V. Solé, Europhysics Letters 60, 4 (2002).

[14] F. Papadopoulos, M. Kitsak, M. A. Serrano, M. Boguñá, D. Krioukov, Nature 489, 537-540 (2012).

[15] A. L. Barabási, Nature 489, 507-508 (2012).

[16] V. Cutello, G. Narzisi, G. Nicosia, J. Royal Soc. Interface, 3, 139-151 (2006).

[17] A. Patanè, A. Santoro, J. Costanza, G. Nicosia, IEEE Trans. on Biomedical Circuits and Systems, 9, 555-571 (2015).

[18] E. Bullmore, O. Sporns, Nat. Rev. Neurosci 10, 186-198 (2009)

[19] V. Nicosia, P. Vertes, R. Shafer, V. Latora, E. Bullmore, Proc. Natl. Acad. Sci. USA bf 110, 7880-7885 (2013).

[20] L. F. Seoane, R. Solé, Phys. Rev. E 92, 032807 (2015).

[21] L. F. Seoane, R. Solé, arXiv:1310.6372 (2015).

[22] O. Shoval, H. Sheftel, G. Shinar, Y. Hart, O. Ramote, A. Mayo, E. Dekel,K. Kavanagh, U. Alon, Science 336, 1157-1160 (2012).

[23] H. Sheftel, O. Shoval, A. Mayo, U. Alon, Ecol. Evol. 3, 1471-1483 (2013).

[24] J. Goñi, A. Avena-Koenigsberger, N.V. de Mendizabal, M. van den Heuvel, R. Betzel, O. Sporns, PLoS ONE 8, e58070 (2013)

[25] A. Avena-Koenigsberger, J. Goñi, R. Betzel, M. van den Heuvel, A. Griffa, P. Hagmann, J. Thiran, O. Sporns, Phil. Trans. R. Soc. B 369, 20130530 (2014).

[26] M. Kivelä, A. Arenas, M. Barthelemy, J. P. Gleeson, Y. Moreno, M. A. Porter, J. Complex Network 2, $203-271$ (2014).

[27] S. Boccaletti et al., Physics Reports 544 1, 1-122 (2014).

[28] M. De Domenico, A. Solé-Ribalta, E. Cozzo, M. Kivelä, Y. Moreno, M. A. Porter, S. Gómez, A. Arenas, Phys. Rev. X 3(4), 041022 (2013).

[29] F. Battiston. V. Nicosia, V. Latora, EPJ ST 226(3), 401-416 (2017).

[30] S. V. Buldyrev, R. Parshani, G. Paul, H. E. Stanley, S.
Havlin, Nature 464 1025-1028 (2010).

[31] F. Radicchi, A. Arenas, Nat. Phys. 9, 717-720 (2013).

[32] C. D. Brummitt, G. Barnett, R. M. D'Souza, J. R. Soc. Interface 12, 20150712 (2015).

[33] S. Gómez, A. Díaz-Guilera, J. Gómez-Gardeñes, C. J. Perez-Vicente, Y. Moreno, A. Arenas, Phys. Rev. Lett. 110, 028701 (2013).

[34] V. Nicosia, P. S. Skardal, A. Arenas, V. Latora, Phys. Rev. Lett. 118, 138302 (2017).

[35] M. Diakonova, V. Nicosia, V. Latora, M. San Miguel, New J. Phys. 18, 023010 (2016).

[36] F. Battiston, V. Nicosia, V. Latora, M. San Miguel, Scientific Reports 7, 1 (2017).

[37] J. Sanz, C.Y. Xia, S. Meloni, Y. Moreno, Phys. Rev. X 4, 041005 (2014).

[38] J.P. Gleeson, K.P. O'Sullivan, R.A. Baños, Y. Moreno, Phys. Rev. X 6, 021019 (2016).

[39] L. Lacasa, M. Cea, M. Zanin, Physica A 388, 3948-3954 (2009)

[40] P. Fleurquin, J. J. Ramasco, V. M. Eguiluz, Scientific Reports 3, 1159 (2013).

[41] A. Cook, H.A.P. Blom, F. Lillo, R. N. Mantegna, S. Miccichè, D. Rivas, R. Vázquez, M. Zanin, JATM 42, 149158 (2015).

[42] V. Colizza, A. Barrat, M. Barthelemy, A. Vespignani, Proc. Natl. Acad. Sci. U.S.A. 103, 2015 (2006).

[43] M. F. C. Gomes, A. Pastore y Piontti, L. Rossi, D. Chao, I. Longini, M. E. Halloran, A. Vespignani, PLOS Currents Outbreaks, 2014 (2014).

[44] V. Nicosia, G. Bianconi, V. Latora, M. Barthelemy, Phys. Rev. Lett. 111, 058701 (2013).

[45] J.Y. Kim, K.-I. Goh, Phys. Rev. Lett. 111, 058702 (2013).

[46] V. Nicosia, G. Bianconi, V. Latora, M. Barthelemy, Phys. Rev. E 90, 042807 (2014).

[47] K. Miettinen Kluwer Academic Publishers 12, (1999).

[48] K. Deb, Springer US, 273-316 (2005)

[49] K. Deb, John Wiley 85 Sons (2001).

[50] A. Barrat, M. Barthélemy, R. Pastor-Satorras, A. Vespignani, Proc. Natl. Acad. Sci. U.S.A. 101, 3747 (2004).

[51] R. Guimera, S. Mossa, A. Turtschi, L.-A.-N. Amaral, Proc. Natl. Acad. Sci. U.S.A. 102, 7794 (2005).

[52] M. Zanin, F. Lillo, Eur. Phys. J. Special Topics 215, 5-21 (2013).

[53] T. Verma, N. A. M. Araújo, H. J. Herrmann, Scientific Reports 4, 5638 (2014).

[54] A. Cook, Ashgate Publishing Limited, England (2016).

[55] A. Cardillo, J. Gómez-Gardeñes, M. Zanin, M. Romance, D. Papo, F. del Pozo, S. Boccaletti, Scientific Reports 3, 1344 (2013).

[56] A. Cardillo, M. Zanin, J. Gómez-Gardeñes, M. Romance, A. J. García del Amo, S. Boccaletti, Eur. Phys. J. Spec. Top. 215, 23 (2013).

[57] V. Nicosia, V. Latora, Phys. Rev. E 92, 032805 (2015).

[58] A. Santoro, V. Latora, G. Nicosia, V. Nicosia, Supplementary material to "Pareto optimality in multilayer networks".

[59] F. Battiston, V. Nicosia, V. Latora, Phys. Rev. E 89, 032804 (2014).

[60] G. Bianconi, Phys. Rev. E 87, 062806 (2013).

[61] J. Tinbergen Twentieth Century Fund, New York (1962).

[62] P. Pöyhönen Weltwirtschaftliches Archiv. 90, 93-100 (1963).

[63] W. K. Hastings Biometrika 57, 1, 97-109 (1970). 
[64] T. W. Anderson, Ann. Math. Statist. 33(3), 1148-1159 (1962).

[65] G. K. Zipf, Human behavior and the principle of least effort, Addison-Wesley Press (1949).

[66] M. Batty, Nature 444(7119), 592-596 (2006).

[67] P. Erdös, A. Rényi, s, 2010. Publ. Math.-Debrecen 6, 290 (1959).

[68] K. Deb, A. Pratap, S. Agarwal, T. Meyarivan IEEE Transactions on Evol. Computation 6, 182-197 (2002).

[69] E. Zitzler, L. Thiele Proc. of the $5^{\text {th }}$ Intern. Conf. on Parallel Problem Solving from Nature 292-304 (1998).

[70] E. Zitzler, L. Thiele, M. Laumanns, C. M. Fonseca, and V. Grunert da Fonseca. IEEE Transactions on Evolutionary Computation, 7(2), 117-132 (2003).

[71] http://data.worldbank.org/indicator/NY.GDP.PCAP.CD (Accessed: 1 December 2017) 


\section{SUPPLEMENTARY MATERIAL}

\section{HYPERVOLUME INDICATOR}

A commonly used measure to quantitatively assess the quality of a Pareto front in multi-objective optimization theory is the hypervolume indicator $[1,2]$, which is often referred in literature as 'size of the space covered' by the Pareto front or 'S-metric'. Given a set of Pareto optimal points $\mathcal{P}=\left\{\left(G_{1}, F_{1}\right), \ldots,\left(G_{n}, F_{n}\right)\right\}$ in the F-G plane, the hypervolume indicator $I_{H}(\mathcal{P})$ gives the area enclosed by the union of the rectangles defined by each of the points and $\left(G_{i}, F_{i}\right)$ in $\mathcal{P}$ and a reference point $\left(G_{r e f}, F_{\text {ref }}\right)$. Canonical choices for the reference point are either one of the points that optimizes each cost function alone (also called ideal points $[3,4]$ ) or the worst point, i.e., the point corresponding to the worst possible values of the two functions to be optimized. We chose the worst point, which in our case is the point of coordinates $\left(G_{\max }, F_{\min }\right)$. In the paper we defined a normalized hypervolume indicator to compare the distance of different transportation networks with respect to their theoretical Pareto front, which is obtained through a multi-objective optimization algorithm [5]. The metric is defined as $\Delta_{H}=\left|I_{H_{\text {pobs }}}-I_{H_{\mathcal{p}_{\text {th }}}}\right| /\left(I_{H_{\mathcal{P}^{\text {th }}}} K\right)$, where $I_{H_{\text {pobs }}}$ and $I_{H_{\mathcal{p}_{\text {th }}}}$ respectively represent the hypervolume indicator of the observed and of the theoretical Pareto front, while $K$ is the total number of routes in the multiplex. Notice that, since $\Delta_{H}$ is a normalized relative error, the choice of the reference point has little impact on its actual value. We interpreted $\Delta H$ as a measure of the potential improvement of a system towards its theoretical Pareto front.

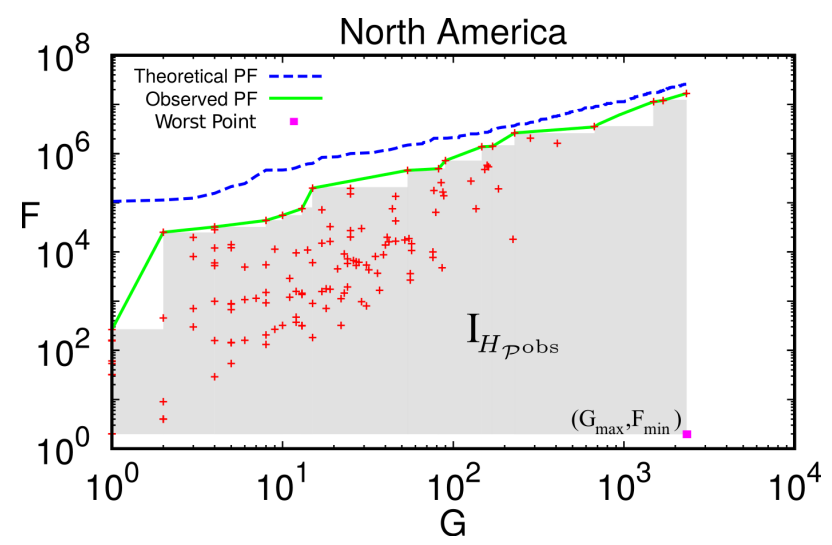

FIG. 5: (Color online) Hypervolume indicator of the observed Pareto front of the North American airlines computed with respect to the reference point $\left(G_{\max }, F_{\min }\right)$

\section{TRANSPORTATION NETWORK DATASETS}

The multiplex transportation systems we considered in the main text are: (i) the undirected routes of the six continental airlines (OpenFlight), (ii) the UK national railway network, and (iii) the UK national coach network. These datasets represent the main modes of public transportation networks. We describe below these data sets and summarize in Table I the basic structural properties of the corresponding multiplex networks. Additional details for the continental multiplex airlines can be found in [7] and the original dataset can be downloaded at [8], while the other two datasets are available for download at [9]. We considered all these multiplexes as unweighted.

\begin{tabular}{|c|c|c|c|}
\hline Multiplex & $\mathrm{N}$ & $\mathrm{M}$ & $\left\langle N^{[\alpha]}\right\rangle$ \\
\hline Africa & 238 & 84 & 9.8 \\
Asia & 795 & 213 & 24.4 \\
Europe & 593 & 175 & 21.8 \\
North America & 1029 & 143 & 24.9 \\
Oceania & 261 & 37 & 14.1 \\
South America & 300 & 58 & 15.1 \\
UK Coaches & 11738 & 1207 & 16.6 \\
UK Trains & 1658 & 41 & 64.27 \\
\hline
\end{tabular}

TABLE I: The number of nodes $N$, number of layers $M$ and the average layer activity $\left\langle N^{[\alpha]}\right\rangle$ are reported for the eight multiplexes analyzed in this work.

OpenFlight - The networks of aerial routes originally used in [7] were constructed from the collaborative free online tool OpenFlight [6], which allows to map flights all around the world. For each route, the dataset contains information about the staring airport, the destination airport, and the company which operates each flight. Six different multiplex networks were constructed, one for each continent (Africa, Asia, Europe, North America, Oceania, South America), each consisting of as many layers as airlines operating in that continent. The edges on each layer are the direct routes operated by the corresponding airline, and the active nodes on each layer are the airports that are connected by at least one route operated by that company.

OpenTrainTimes - The timetable extracted from OpenTrainTimes [10] contain real-time information about the routes operated by 41 different railway companies over 2944 stations across the UK. In this case, nodes represent railway stations, while a link exists between two nodes only if there is at least one service between them. The routes of OpenTrainTimes are regularly updated using the real-time data feeds system provided by the official network rail website [11]. For each route, we have information about the starting station, the end station, the company which operates the service, and also the days in which they operate their routes. For the analysis 
done in the main text, we have constructed a multiplex network with 1658 nodes (the stations were aggregated at the city level) and 41 layers representing the different companies.

UK coaches - We used the UK coach data set available at Ref. [12], which includes data from the National Coach Services Database (NCSD). The dataset contains information about 17433 routes between 12767 locations operated by 1219 different regional operators. In this case, nodes represent coach stations or stops, while a link exists between two nodes only if there is at least one connection between them. Layers represent the different regional service providers. Notice that for each route we have information about the start, middle and end points, as well as the regional operator code. Starting from this dataset we constructed a multiplex network having 1207 regional service providers (layers) operating over 11738 stops (nodes). The analysis does not include providers operating circular services.

\section{DETAILS ON THE MMOO MODEL}

In the main text, we proposed the Multiplex MultiObjective Optimization (MMOO) model where the probability to attach a new edge between two nodes at a given time step is given in Eq. (1). It is important to notice that such a probability also depends on two non-negative constants $c_{1}$ and $c_{2}$, which allow to have a non-zero probability when creating a new edge to a node that is isolated at all the existing layers. We compared the synthetic and empirical distributions for the eight transportation systems with $c_{1}=1$ and $c_{2}=1$. In Table II we report the p-values of the corresponding two-sample Cramer-vonMises test.

For most of the multiplex analyzed, the MMOO model with $c_{1}=c_{2}=1$ performs quite well for three different structural metrics. In particular, we find that the synthetic and empirical distributions of layer activity $N^{[\alpha]}$ and node total degree $o_{i}$ are indistinguishable. Similarly, we obtain meaningful statistical evidence (but a lower p-value) for the distribution of edge overlap $o_{i j}$.

To further evaluate the dependence of $c_{1}$ and $c_{2}$ on the model, we performed extensive Monte Carlo simulations where the two constants $c_{1}, c_{2}$ were tuned in the range $(0,10)$ with steps of 0.1 .

In Table we report, for each system, the values of $c_{1}$ and $c_{2}$ for which the two-sample Cramer-von-Mises test provides the best (lowest) p-values for the distributions of node total degree $o_{i}$, edge overlap $o_{i j}$, layer activity $N^{[\alpha]}$, node activity $B_{i}$ and pairwise inter-layer correlation $H_{\alpha, \beta}$ (see Ref. [7] for details). For the sake of clarity, we also report in Fig. 6 the placement of each airline in the efficiency-competition plane and the corresponding Pareto front for the four continental airlines of Asia, Europe, South America and Oceania. In each plot we

\begin{tabular}{|c|ccccc|}
\hline Multiplex & $o_{i}$ & $o_{i j}$ & $N^{[\alpha]}$ & $B_{i}$ & $H_{\alpha, \beta}$ \\
\hline Africa & $<0.01$ & 0.15 & $<0.01$ & 0.2 & 0.2 \\
Asia & $<0.01$ & 0.5 & $<0.01$ & 0.05 & 0.05 \\
Europe & $<0.01$ & $<0.01$ & $<0.01$ & 0.05 & 0.025 \\
North America & $<0.01$ & 0.5 & $<0.01$ & 0.25 & 0.75 \\
Oceania & 0.1 & 0.15 & $<0.01$ & 0.75 & $<0.01$ \\
South America & $<0.01$ & 0.75 & $<0.01$ & 0.1 & 0.25 \\
UK Coaches & $<0.01$ & $<0.01$ & $<0.01$ & 0.75 & 0.75 \\
UK Trains & $<0.01$ & $<0.01$ & $<0.01$ & 0.5 & 0.05 \\
\hline
\end{tabular}

TABLE II: List of the p-values obtained for the two-sample Cramer-von-Mises test when comparing the synthetic and empirical distributions of the eight transportation systems analyzed in the main text. Five different structural measures were tested, namely, the node overlap $\left(o_{i}\right)$, the edge overlap $\left(o_{i j}\right)$, the layer activity $\left(N^{[\alpha]}\right)$, node activity $\left(B_{i}\right)$ and the normalized Hamming distance $\left(H_{\alpha, \beta}\right)$ [7]. Notice that we used $c_{1}=1, c_{2}=1$ in the MMOO model for obtaining the synthetic distributions.

highlighted the top ten companies by number of passengers in 2012. Notice that whenever an airline group is listed in the top ten airways, we report each element of the group.

\begin{tabular}{|c|ccccc|c|c|}
\hline Multiplex & $o_{i}$ & $o_{i j}$ & $N^{[\alpha]}$ & $B_{i}$ & $H_{\alpha, \beta}$ & $c_{1}$ & $c_{2}$ \\
\hline Africa & $<0.01$ & $<0.01$ & $<0.01$ & 0.1 & 0.25 & 0.2 & 9.3 \\
Asia & $<0.01$ & $<0.01$ & $<0.01$ & $<0.01$ & 0.1 & 0.2 & 3.0 \\
Europe & $<0.01$ & $<0.01$ & $<0.01$ & $<0.01$ & 0.025 & 1.0 & 8.2 \\
North America & $<0.01$ & $<0.01$ & $<0.01$ & 0.1 & 0.75 & 0.1 & 9.0 \\
Oceania & 0.025 & 0.05 & $<0.01$ & 0.75 & $<0.01$ & 1.8 & 6.0 \\
South America & $<0.01$ & $<0.01$ & $<0.01$ & 0.1 & 0.5 & 0.2 & 9.0 \\
UK Coaches & $<0.01$ & $<0.01$ & $<0.01$ & 0.5 & 0.75 & 4.0 & 6.5 \\
UK Trains & $<0.01$ & $<0.01$ & $<0.01$ & 0.25 & $<0.01$ & 2.1 & 5.3 \\
\hline
\end{tabular}

TABLE III: Values of $c_{1}$ and $c_{2}$ for which we obtain the best (lowest) p-values of the Cramer-von-Mises test for all the five structural multiplex metrics.

[1] E. Zitzler, L. Thiele Proc. of the $5^{\text {th }}$ Intern. Conf. on Parallel Problem Solving from Nature 292-304 (1998).

[2] E. Zitzler, L. Thiele, M. Laumanns, C. M. Foneseca, V. Grunert da Fonseca. IEEE Transactions on Evolutionary Computation 7(2):117-132, (2003).

[3] K. Miettinen Kluwer Academic Publishers 12, (1999).

[4] K. Deb, Springer US, 273-316 (2005)

[5] K. Deb, A. Pratap, S. Agarwal, T. Meyarivan IEEE Transactions on Evol. Computation 6, 182-197 (2002).

[6] http://openflights .org/

[7] V. Nicosia, V. Latora, Phys. Rev. E 92, 032805 (2015).

[8] http://www.maths.qmul.ac.uk/ vnicosia/sw.html

[9] http://github.com/andresantoro/tranet_data/

[10] http://www.opentraintimes.com/

[11] http://www.networkrail.co.uk/

[12] http://www.travelinedata.org.uk/ 

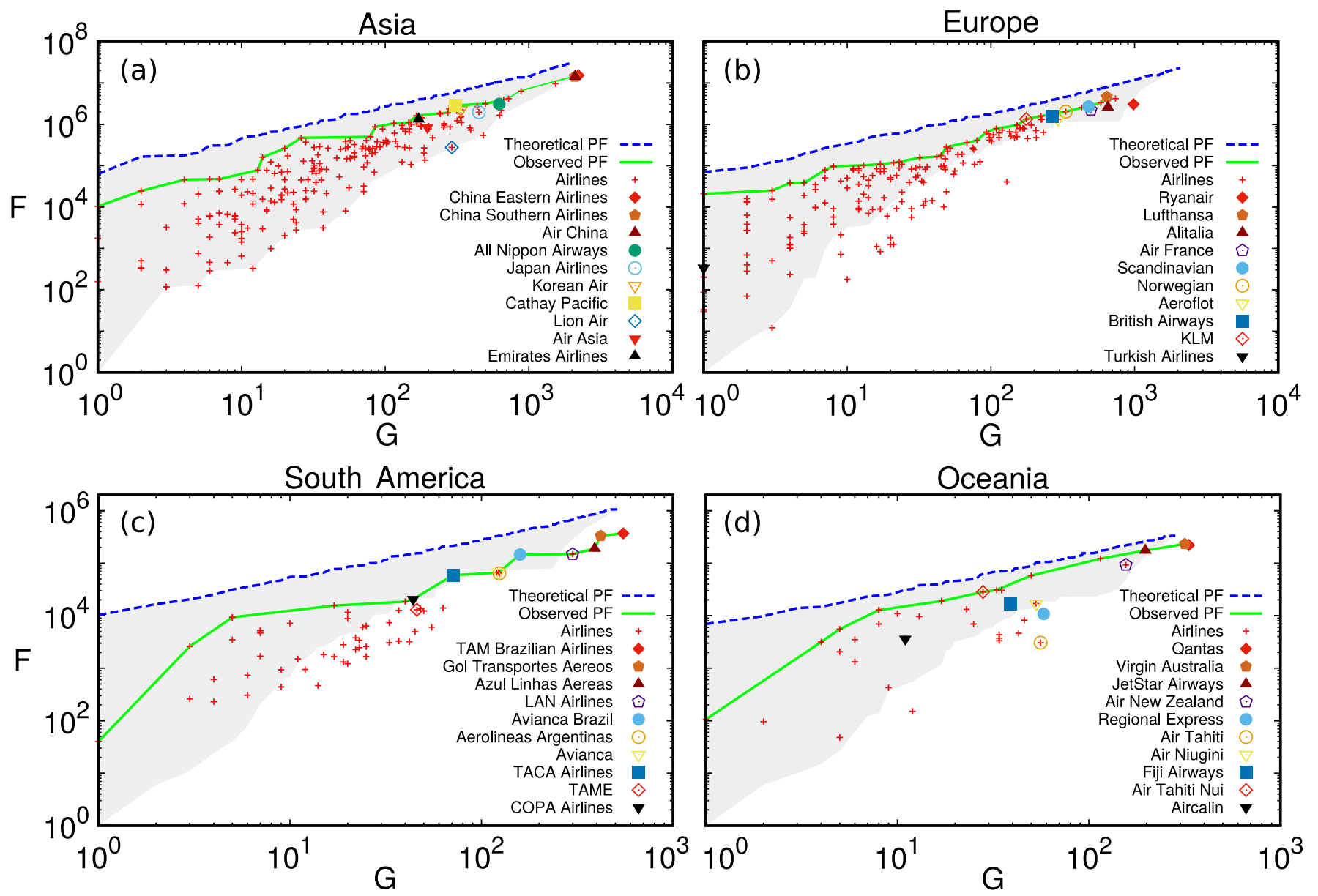

FIG. 6: (Colour online) The observed Pareto front (solid green line) along with the top ten airlines by number of passengers for Asia (a), Europe (b), South America (c), and Oceania (d). For each continent, the Theoretical Pareto front (dashed blue line) is obtained by considering the non-dominated points of $10^{5}$ realizations of the MMOO model (the range of variability obtained through the simulations is indicated by the shaded gray region). Notice that in almost all the cases, the top airlines of a continent belong to the observed Pareto front. 